\title{
ESTUDIO SOBRE DISTRIBUCIÓN GEOGRÁFICA E IDENTIFICACIÓN DE HOSPEDEROS DE LA MOSCA DEL MEDITERRÁNEO EN OCHO CANTONES DE MANABÍ. ECUADOR
}

\author{
STUDY ON THE GEOGRAPHIC DISTRIBUTION AND HOST \\ IDENTIFICATION OF THE MEDITERRANEAN FRUIT FLY IN EIGHT \\ DISTRICTS OF MANABÍ. ECUADOR
}

\author{
Carlos Oswaldo Valarezo Beltrón, Mg. \\ Docente ESPAM \\ cvalarezo@espam.edu.ec \\ Gonzalo Oswaldo Valarezo Cely, MSc, UTM \\ Clotilde Andrade Varela, MSc, UPSE \\ Néstor Valarezo Beltrón, Ing. Agro. INIAP
}

\section{RESUMEN}

Los objetivos de esta investigación fueron determinar la distribución geográfica de C.capitata y la identificación de sus frutales hospederos para la determinación de acciones pertinentes por los organismos competentes. El trabajo se realizó desde agosto del 2001 hasta abril del 2002, en los cantones Portoviejo, Santa Ana, Chone, Junín, Bolívar, Tosagua, Rocafuerte y Sucre. Para el manejo experimental se recurrió a las técnicas de trampeo de moscas y muestreo de frutos en cada una de las 68 localidades elegidas. El trampeo se sustentó en el empleo de trampas tipo Jackson con trimedlure (atrayente sexual para machos adultos) y trampas tipo McPhail con torulas (atrayente alimenticio a base de levaduras que atrae a machos y hembras). El muestreo consistió en recolectar frutos del árbol y del suelo, para finalmente procesarlos en el laboratorio y esperar la emergencia de los adultos. Los resultados mostraron que según el trampeo, en la provincia de Manabí; C.capitata se encuentra localizada sólo en cinco parroquias rurales del cantón Portoviejo (Colón, Calderón, Picoazá, Riochico y Crucita). Los frutos hospederos de la mosca del Mediterráneo identificados en Manabí (Portoviejo) fueron almendro (T.catappa), guayaba (P.guajaba) y pechiche (V. gigantea). Los meses con incidencia de C.capitata resultaron agosto, septiembre, noviembre, enero, febrero y marzo.

Palabras clave: distribución, hospederos, mosca, mediterráneo.

\begin{abstract}
The objectives of this research were to determine the geographical distribution of C.capitata and identifying its host fruit for the determination of appropriate action by the competent authorities. The study was conducted from August 2001 to April 2002 in Portoviejo, Santa Ana, Chone, Junín, Bolivar, Tosagua, Sucre and Rocafuerte cantons. For the experimental management techniques fly trapping and fruit sampling in each of the 68 locations chosen were used. Trapping was based on the use of traps Jackson type with trimedlure (sexual attractant for adult males) and McPhail traps with swabs (based food attractant which attracts yeast males and females). The sample consisted of collecting fruits of the tree and the soil, and finally process them in the laboratory and expect the emergence of adults. The results showed that by trapping in the province of Manabi; C.capitata is located only five rural parishes of the canton Portoviejo (Columbus, Calderon Picoaza, Riochico and Crucita). The host fruit fly Mediterranean identified in Manabi (Portoviejo) were almond (T.catappa), guava (P.guajaba) and pechiche (V. gigantea). The incidence of C.capitata months were August, September, November, January, February and March.
\end{abstract}

Key words: distribution, hosts, fly, mediterranean.

Recibido: enero 2015 Aprobado: abril 2015

\section{Introducción}

A nivel mundial la mosca del Mediterráneo o mosca med C.capitata (Díptera: Tephritidae) es considerada como la plaga más destructiva de la agricultura, ya que infesta más de 260 frutos vegetales y nueces 
causando rechazo en el consumo, exportación y agroindustria. Esta especie es capaz de destruir totalmente la pulpa de las frutas atacadas debido a la presencia del estado larval de este insecto cuyo desarrollo lo realiza en su interior provocando pudriciones o caída, y por lo tanto pérdidas económicas. Esta mosca en América del Norte se ha presentado en California, Florida y Texas donde ha sido erradicada. En América Central se lo encuentra desde México a Guatemala. En América del Sur está presente en Perú, Brasil, Ecuador y Colombia. En Chile la consideran erradicada (Esparza, 1999). En nuestro país ingresó por la frontera sur en 1976 (Molinero, 1984).

En la actualidad el enorme potencial de la región Litoral con fines de exportación de frutales se ve amenazada por la presencia de $\mathrm{C}$. capitata ya que las mismas son severamente vigiladas por los países compradores para evitar el ingreso de la plaga a sus plantaciones. Otro riesgo que se presenta es la posibilidad de que por controlarla se empleen productos químicos cuya residualidad y toxicidad tampoco está permitida por los compradores por el peligro que representa para la salud de los consumidores.

Según datos estadísticos del Ministerio de Agricultura y Ganadería en Manabí el área sembrada en el 2001 de varias especies de frutales que potencialmente serían afectados por el ataque de C. capitata son; mandarina 7200 ha., mango 420 ha., naranja 13100 ha. y toronja 3600 ha. Existe alguna información sobre los principales hospederos de esta mosca en el país, pero en Manabí no se tienen datos, por lo que se necesita realizar estudios básicos sobre este insecto-plaga, como son la identificación de sus principales hospederos y su distribución geográfica

\section{Objetivos}

- Determinar la distribución geográfica de C. capitata en 8 cantones de Manabí.

- Identificar los frutales hospederos de C.capitata, de importancia económica.

\section{Metodología}

El área de este trabajo se ubicó al norte, en el cantón Chone, al sur en Santa Ana y al oeste Portoviejo (Las Gilces) abarcando además los cantones Bolívar, Junín, Tosagua, Sucre y Rocafuerte; influenciados por las cuencas hidrográficas de los ríos Chone, Carrizal, Chico y Portoviejo.Mientras que los trabajos de campo y laboratorio se cumplieron desde el mes de Agosto del 2001 hasta Abril del 2002, para lo cual se realizaron 18 visitas quincenales para el monitoreo de C. capitata con los atrayentes sexuales y alimenticios en todas y en cada una de las 68 localidades. Paralelamente se tomaron muestras de frutos de 22 especies, en este caso de acuerdo a la época de producción o cosecha de los respectivos frutos, los mismos que fueron procesados en el laboratorio de Entomología de la Universidad Técnica de Manabí. Para cumplir con el manejo experimental, se recurrió al trampeo y muestreo de frutos en cada una de las localidades elegidas. Esta actividad se sustentó en el uso de trampas Jackson, con el atrayente sexual trimedlure que atrae a machos adultos de C capitata y Mcphail con el atrayente alimenticio torulas, para machos y hembras de la mosca med, las mismas que se distribuyeron en las localidades descritas. En este caso se registró el número de machos adultos/trampa Jackson y número de machos y hembras/trampa McPhail.

Muestreo de frutos,- Esta labor consistió en la recolección de frutos, tanto en el árbol como en el suelo alrededor de éste. Las finitas colectadas en el árbol tuvieron 3/4 de su madurez y se seleccionaron de 5-10 frutos por árbol. Para colectar finitos del suelo, éstos no debían presentar signos de descomposición. Durante la prueba se tomaron muestras de frutos de 22 especies frutícolas (guayaba, anona, café, mandarina, naranja, fruta china, fruta del pan, toronja, mamey colorado, mango, almendro, frutillo, guanábano, achochilla, granada, marañón, cereza, ovo, pechiche, mamey Cartagena, níspero y arazá), los mismos que fueron colocados en cámaras de cría hasta la emergencia de los adultos de C. capitata.

\section{Resultados y discusión}

Las condiciones climáticas en el área con presencia de C capitata variaron entre $311 \mathrm{~mm}$ de precipitación anual en la parroquia Crucita y 732mm en Portoviejo, medias nauales de temperatura y humedad relativa de $25,4^{\circ} \mathrm{C}$ y $80 \%$ respectivamente. La altitud de las localidades estuvo entre 3 y $130 \mathrm{msnm}$ en Las Gilces y San Felipe, respectivamente.

Durante las 18 evaluaciones en trampas Jackson en cada uno de los cantones considerados en el presente estudio, se capturaron 44 individuos de C. capitata, todos ellos en trampas ubicadas en localidades pertenecientes al cantón Portoviejo, cuya distribución se puede observar en la tabla 1 , en donde el mayor número (24) cayeron en el sitio Pimpiguasí (parroquia Calderón) siguiéndole El Limón (parroquia Colón) con 13, y Las Gilces (parroquia Crucita) tuvo 7 en dos trampas. 
Tabla 1. Número de machos adultos de C.capitata capturados en trampas Jackson en varios sitios del cantón Portoviejo. Portoviejo 2002.

\begin{tabular}{|lc|}
\hline Localidad & No de adultos $^{\circ}$ \\
\hline Limón, Colón. & 13 \\
Las Gilces, Crucita. & 2 \\
Las Gilces, Crucita. & 5 \\
Pimpiguasi, Calderón. & 24 \\
TOTAL & 44 \\
\hline
\end{tabular}

En la tabla 2 se presenta el resultado de las evaluaciones de adultos de C.capitata en trampas McPhail, totalizando 49 individuos 16 machos y 33 hembras, todos también en Portoviejo, ya que en los demás cantones no se registraron poblaciones del insecto. Este dato permite establecer como relación que por cada macho hay dos hembras. Las localidades donde se capturó el mayor número de adultos fue nuevamente Pimpiguasí con 17 individuos. El Limón y Mejía (parroquia Picoazá) tuvieron 12 cada uno, en Las Gilces hubo 7 y San Felipe (parroquia Riochico) solo 1 macho.

En la tabla 3 se observa la distribución mensual de las poblaciones de adultos de C.capitata capturados en el cantón Portoviejo, en los dos tipos de trampa utilizados. Las mayores poblaciones se concentran en los meses de enero, febrero y marzo, por el contrario en abril y octubre desaparecen y en los restantes meses el número de adultos es bastante bajo coincidiendo con la falta de frutos hospederos en esa época. En este caso hay concordancia con la dinámica poblacional de A.fraterculus en Manabí y Guayas (Arias et al, 2001).

De manera general, se puede asumir que de acuerdo al tipo de trampa, los dos demostraron la misma capacidad en cuanto al número de individuos capturados así como similitud con respecto a la época en que sucedieron las capturas.

Tabla 2. Número de adultos de C.capitata capturados en trampas Mcphail en varios sitios del cantón Portoviejo 2002.

\begin{tabular}{|l|c|c|c|}
\hline \multicolumn{3}{|c|}{ Localidad } & \multicolumn{3}{|c|}{ Adultos } \\
\hline & Machos & Hembras & Total \\
\hline Limón, Colón & 2 & 10 & 12 \\
\hline San Felipe, Riochico & 1 & 0 & 1 \\
\hline Las Gilces, Crucita & 3 & 4 & 7 \\
\hline Pimpiguasi, Calderón & 8 & 9 & 17 \\
\hline Mejia,Picoazá & 2 & 10 & 12 \\
\hline TOTAL & 16 & 33 & 49 \\
\hline
\end{tabular}

Tabla 3. Número de adultos de C.capitata capturados en varias localidades del cantón Portoviejo (2001- 2002).

\begin{tabular}{|lccc|}
\hline Mes & Trampa Jackson & Trampa McPhail & Total \\
\hline Agosto & 2 & 0 & 2 \\
Séptiembr & 2 & 4 & 6 \\
Octubre & 0 & 0 & 0 \\
Noviembre & 2 & 0 & 2 \\
Diciembre & 0 & 1 & 1 \\
Enero & 17 & 19 & 36 \\
Febrero & 17 & 12 & 29 \\
Marzo & 5 & 13 & 18 \\
Abril & 0 & 0 & 0 \\
Total & 45 & 49 & 94 \\
\hline
\end{tabular}

En cuanto a la identificación de hospederos de C. capitata con muestras de 22 frutos diferentes provenientes de las cantones en estudio; se logró determinar que sólo las muestras pertenecientes a Portoviejo presentaron emergencia de adultos machos y hembras, corroborando los datos obtenidos en las trampas, pero sólo en tres especies de frutales por lo cual se determinó su condición de hospederos: Almendro (T.catappa). Guayaba (P.guajaba) y Pechiche (V. gigantea). Al respecto merece comentar que por ahora estas especies tienen poca o ninguna importancia económica. Aparentemente responde al predominio que ejerce el género Anastrephasobre frutales de mayor importancia en nuestro medio, lo que provoca un desplazamiento de $\mathrm{C}$. capitata a los tres hospederos identificados

Finalmente podemos mencionar que por ahora C.capitata está limitada al cantón Portoviejo, aunque no se descarta que esté en otras zonas urbanas o turísticas de la provincia como Manta, Bahía, Pto. López, etc. debido al traslado masivo de visitantes y comerciantes de la Sierra en ciertas épocas del año, los cuales pueden trasladar frutos hospederos infestados con C.capitata, ya que en esa región del país está mayormente diseminada y desde mucho antes (Jirón, 2000). Las poblaciones de C.capitata a más de estar focalizadas, aún están en poblaciones bastante bajas si se compara con la abundante presencia de varias especies del género Anastrepha, entre las que sobresale nítidamente A. fraterculus. Sin embargo C.capitata por ser plaga cuarentenaria en otros países, puede limitar el potencial exportable de frutos del Ecuador, al disponerse cada vez de menos zonas "libres" de este insecto; requisito exigido por los importadores de frutas.

\section{Conclusiones}

1. En la provincia de Manabí C.capitata está localizada solamente en el cantón Portoviejo.

2. Las parroquias de Portoviejo donde ha sido encontrada la mosca de la fruta son; Colón, Calderón ,Pícoazá , Ríochico y Crucita.

3. Los frutos hospederos de la mosca del Mediterráneo identificados en Manabi (Portoviejo) son almendro, guayaba y pechiche.

4. Los meses con mayor incidencia de C. capitatafueron ; Enero, Febrero y Marzo.

5. Las feromonas (trampas Jackson) y los atrayentes alimenticios (Trampas Mcphail) son eficientes para la captura de adultos de C. capitata.

\section{Recomendaciones}

A partir de los resultados investigativos que se muestran, se deberá dar continuidad a otros es- 
tudios en las zonas aledañas, donde se encuentra este insecto plaga.

Que los organismos competentes (GAD's, MAGAP, Agrocalidad, entre otros), tomen las medidas pertinentes para el control y prevención de la plaga.

\section{Bibliografía}

Arias, M.; Jines, A.; Carrera, C. 2001. Dinámica poblacional de Anastrephafraterculus (Diptera: Tephritidae) y sus principales hospederos. In. memorias XI seminario de sanidad vegetal(nov 20-23/2001). Universidad técnica de Babahoyo. Babahoyo, EC. p 1-53.

Esparza, J. 1999. El Programa Chile-Perú contra las Moscas de las Frutas. Revista Comunica 4(11):814.

INIAP (Instituto Nacional Autónomo de Investigaciones Agropecuarias, EC). 2001.Proyecto generación de alternativas tecnológicas para el control de mosca de la fruta en el litoral ecuatoriano; informe anual. Quito, EC. 45 p.

Jirón, L. 2000. Manejo Integrado de la Mosca de la Fruta. Revista Cultivos Controlados 2(3);18-19.

Molineros, J. 1984. La mosca de la fruta en el Ecuador. In. I Encuentro Entomológico Ecuatoriano. Dic 1984. 31-32. 